\title{
Electrostatic interactions in the
} association of proteins: An analysis of the thrombin-hirudin complex

\author{
ANDREJ KARSHIKOV, ${ }^{1}$ WOLFRAM BODE, ${ }^{1}$ ALEXANDER TULINSKY,${ }^{2}$ \\ AND STUART R. STONE ${ }^{3}$ \\ ${ }^{1}$ Max-Planck-Institut für Biochemie, D-8033 Martinsried, Germany \\ ${ }^{2}$ Department of Chemistry, Michigan State University, East Lansing, Michigan 48824-1322 \\ ${ }^{3}$ Department of Haematology, University of Cambridge, MRC Centre, Hills Road, Cambridge CB2 2QH, UK \\ (Received November 1, 1991; Revised Manuscript Received February 5, 1992)
}

\begin{abstract}
The role of electrostatic interactions in stabilization of the thrombin-hirudin complex has been investigated by means of two macroscopic approaches: the modified Tanford-Kirkwood model and the finite-difference method for numerical solution of the Poisson-Boltzmann equations. The electrostatic potentials around the thrombin and hirudin molecules were asymmetric and complementary, and it is suggested that these fields influence the initial orientation in the process of the complex formation. The change of the electrostatic binding energy due to mutation of acidic residues in hirudin has been calculated and compared with experimentally determined changes in binding energy. In general, the change in electrostatic binding energy for a particular mutation calculated by the modified Tanford-Kirkwood approach agreed well with the experimentally observed change. The finitedifference approach tended to overestimate changes in binding energy when the mutated residues were involved in short-range electrostatic interactions. Decreases in binding energy caused by mutations of amino acids that do not make any direct ionic interactions (e.g., Glu 61 and Glu 62 of hirudin) can be explained in terms of the interaction of these charges with the positive electrostatic potential of thrombin. Differences between the calculated and observed changes in binding energy are discussed in terms of the crystal structure of the thrombin-hirudin complex.
\end{abstract}

Keywords: electrostatic interactions; hirudin; protein-protein interactions; thrombin

Thrombin is a serine protease that plays a central role in blood coagulation. It cleaves fibrinogen to yield fibrin monomers that form the basis of the blood clot. In addition, thrombin activates a number of other proteins involved in coagulation. Thrombin can be distinguished from other serine proteases, such as trypsin, in that it achieves its specificity by using binding sites, called exosites, that are distant from the catalytic center (Fenton, 1988). The crystal structures of thrombin (Bode et al., 1989,1992 ) and its complexes with the polypeptide inhibitor hirudin (Kinemage 1; Grütter et al., 1990; Rydel et al., 1990,1991 ) have helped to delineate an exosite involved in the binding of hirudin. Whereas the first three N-terminal amino acids of hirudin are bound in the active-site

Reprint requests to: Stuart R. Stone, Department of Haematology, University of Cambridge, MRC Centre, Hills Road, Cambridge CB2 $2 \mathrm{QH}$, UK. cleft of thrombin, the C-terminal region binds to a positively charged surface groove called the fibrinogen-recognition exosite. The $\mathrm{C}$-terminal region of recombinant hirudin (rhir) between residues 55 and 65 contains five negatively charged residues, and results from studies using site-directed mutagenesis indicate that each of these negatively charged residues (Asp 55', Glu 57', Glu 58', Glu $61^{\prime}$, and Glu 62') contributes to the stabilization of the complex (Braun et al., 1988a; Betz et al., 1991). In addition, the effect of ionic strength on the interaction between thrombin and hirudin suggests that electrostatic interactions with the C-terminal region of hirudin are important for the rate of complex formation and the stability of the complex (Stone et al., 1989). However, although protein engineering studies indicate that each of the negatively charged residues in the C-terminal region of hirudin makes about the same contribution to binding energy (Braun et al., 1988a; Stone et al., 1989; Betz et al., 1991), 
the crystallographic structures show that only two of the five acidic residues are in close contact with basic groups on thrombin (Kinemage 2; Grütter et al., 1990; Rydel et al., 1990, 1991). Asp 55' makes salt bridges with Lys $149 \mathrm{E}$ and $\mathrm{Arg} 73$, whereas Glu 58' interacts with Arg 77A. In the present paper, a theoretical analysis of the electrostatic interaction between rhir and thrombin has resolved this apparent inconsistency to some extent.

One of the basic problems of the theoretical analysis of the electrostatic properties of proteins is to find a simple and effective approach for description of the complicated solvent-protein dielectric boundary. There are two approaches to this problem: microscopic models that treat the problem at the atomic level and macroscopic models based on a continuum description of the system (Bashford, 1991). In the macroscopic model the protein molecule is treated as a low dielectric cavity immersed in the high permittivity medium of the solvent and the PoissonBoltzmann equations are solved for this system. The oldest macroscopic model of electrostatic interactions in proteins is that of Kirkwood and Tanford (Kirkwood, 1934; Tanford and Kirkwood, 1957). In this approach, the protein molecule is modeled as a sphere, and this allows analytical solution of the Poisson-Boltzmann equations. The charges carried by the titratable groups are defined as point charges situated on the surface of the dielectric cavity. Later, the model was modified by characterizing the charged groups according to their solvent accessibilities in order to describe the local dielectric environment (Shire et al., 1974; Matthew \& Gurd, 1986). An alternative macroscopic approach is the numerical solution of the Poisson-Boltzmann equations. In this case, the protein-solvent dielectric boundary is taken into account explicitly. The most commonly used numerical technique is the finite-difference method (FD) that was first applied to proteins by Warwicker and Watson (1982). This algorithm has been extended for linear (Klapper et al., 1986) and nonlinear (Sharp \& Honig, 1990) solutions of the Poisson-Boltzmann equations.

In the present paper, both the modified TanfordKirkwood (MTK) and FD computational methods have been used to analyze the electrostatic interactions in the thrombin-rhir complex. The availability of experimental data for the binding energy of a variety of site-specific mutants of rhir provides a good opportunity for a detailed study of the role of charge-charge interactions in the formation of protein complexes. Calculation of the electrostatic potential around thrombin and rhir suggested that "electrostatic steering" encourages an initial orientation of the two molecules that leads to productive complex formation. In agreement with the results from protein engineering studies, a significant contribution to binding energy was found even for residues not directly involved in ionic interactions. Whereas good agreement with the experimental data was in general obtained with the MTK method, FD calculations tended to overestimate the effect of a mutation on binding energy when the mutated residue was involved in short-range electrostatic interactions.

\section{Results}

\section{Electrostatic potential contours}

The analysis of the electrostatic potentials surrounding thrombin and rhir was made on the basis of FD calculations. Both thrombin and rhir are characterized by a marked inhomogeneous distribution of electrostatic potential surrounding the molecules. Two regions of particularly high positive electrostatic potential were found for thrombin. One region shown in Figure 1 results from a positively charged surface groove that has been termed the anion-binding exosite or the fibrinogen-recognition exosite (Bode et al., 1989; Rydel et al., 1990). The C-terminal region of rhir is bound to this site that is formed by the surface loops $34-42$ and $67-80$. A second region of even higher positive potential is found on the other side of the molecule and is formed by the segments Arg 233-Lys 240 and Arg 165-Arg 175 together with several other positively charged residues including Arg 93 and Arg 126 (not shown). It has been suggested that this second region corresponds to the heparin-binding site of thrombin (Bode et al., 1991). A region of negative potential is found between these two regions and the active-site cleft of thrombin is located in this region (Fig. 2). Thus, the electrostatic potential around thrombin exhibits a "sandwich" form with two positive poles enclosing a "ring" of negative potential.

The electrostatic potential of rhir is characterized by a dipolar distribution (Fig. 3). The abundance of acidic residues in the C-terminal tail of rhir creates a region of high negative potential, whereas a less marked positive potential is found around the side of the N-terminal core of rhir that is bound to the active-site cleft of thrombin. In the complex, the electrostatic potentials of thrombin and rhir are complementary. The positive potential of rhir created by the N-terminal amino group and the lysyl residues 24 and 47 are imbedded in the negative potential of thrombin (Fig. 2). The negative potential of the C-terminal tail of rhir is juxtaposed with the positive potential of thrombin created by the fibrinogen-recognition exosite (Fig. 1).

\section{Contribution of electrostatic interactions to binding energy $\left(\Delta G_{b}^{e l}\right)$}

The binding energy $\left(\Delta G_{b}\right)$ for rhir has previously been determined at different ionic strengths and estimates for $\Delta G_{b}^{e l}$ were obtained by fitting these data to a modified Debye-Hückel equation (Stone et al., 1989). Theoretical estimates of $\Delta G_{b}^{e l}$ for rhir at several values of ionic strength have been calculated in the present study by the 

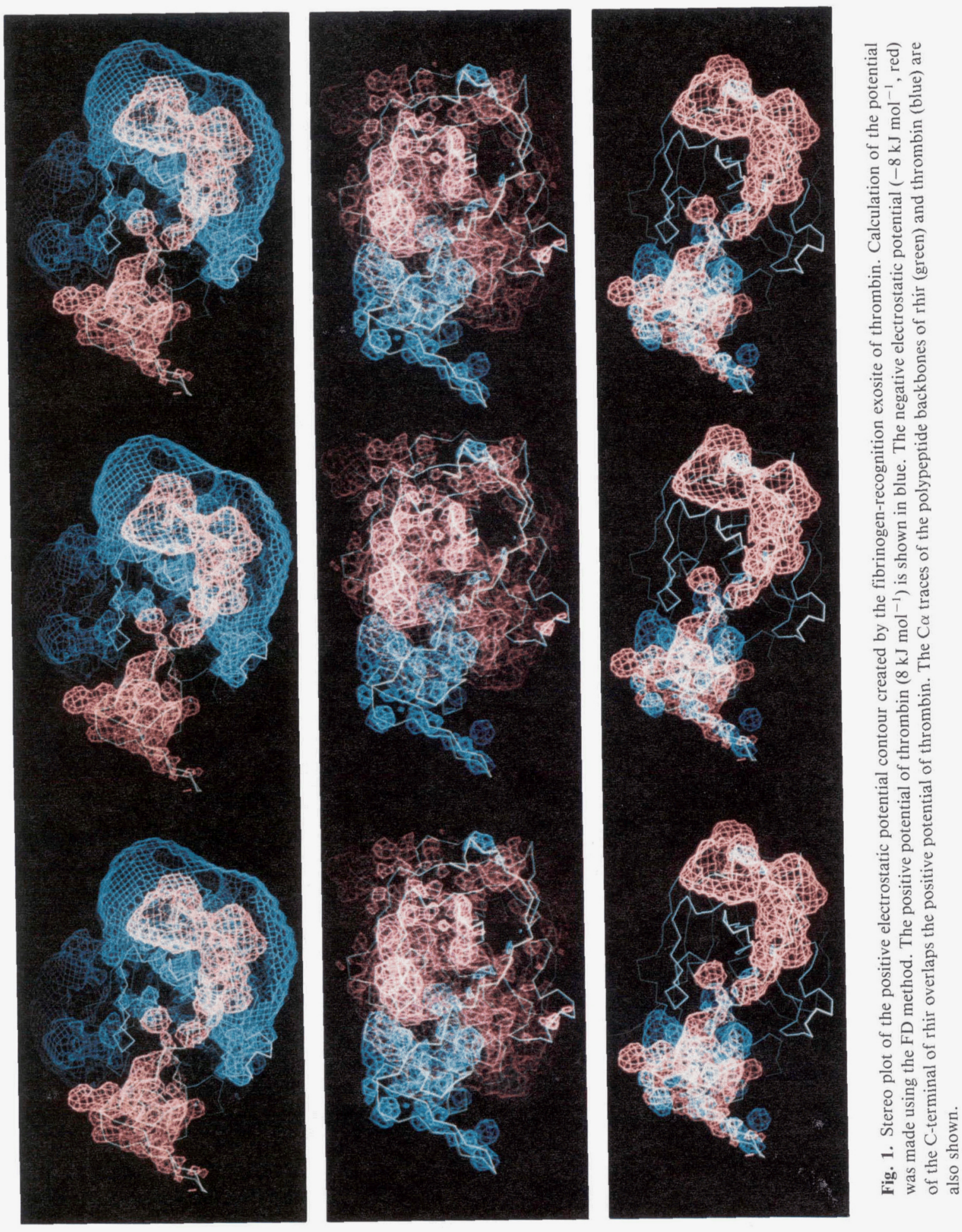

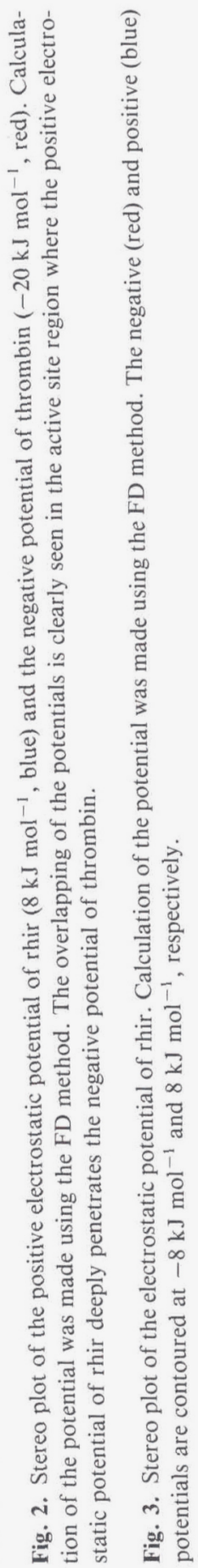


Table 1. Comparison of the experimentally determined binding energy for thrombin-rhir complex with the theoretical contribution of electrostatic interactions to binding energy calculated by the MTK and FD methods ${ }^{\mathrm{a}}$

\begin{tabular}{lcccc}
\hline $\begin{array}{c}I \\
(\mathrm{M})\end{array}$ & $\begin{array}{c}-\Delta G_{b} \\
\left(\mathrm{~kJ} \mathrm{~mol}^{-1}\right)\end{array}$ & $\begin{array}{c}-\Delta G_{b}^{e l}(\mathrm{exp}) \\
\left(\mathrm{kJ} \mathrm{mol}^{-1}\right)\end{array}$ & $\begin{array}{c}-\Delta G_{b}^{e l}(\mathrm{MTK}) \\
\left(\mathrm{kJ} \mathrm{mol}^{-1}\right)\end{array}$ & $\begin{array}{c}-\Delta G_{b}^{e l}(\mathrm{FD}) \\
\left(\mathrm{kJ} \mathrm{mol}^{-1}\right)\end{array}$ \\
\hline 0.0 & 87.2 & 25.0 & 78.5 & 86.2 \\
0.050 & 79.0 & 16.4 & 74.5 & 67.4 \\
0.075 & 77.4 & 15.2 & 74.2 & 61.9 \\
0.125 & 75.0 & 12.8 & 74.0 & 42.3 \\
\hline
\end{tabular}

a The experimentally determined values of $\Delta G_{b}$ are taken from Stone et al. (1989), and experimental estimates of the contribution of electrostatic interactions to binding energy $\left(\Delta G_{b}^{e l}(\exp )\right)$ were calculated from data given in the same paper. Estimates of the electrostatic term of binding energy calculated by the MTK and FD methods are represented by $\Delta G_{b}^{e l}$ (MTK) and $\Delta G_{b}^{e l}(\mathrm{FD})$, respectively.

MTK and FD methods and are given in Table 1. Both methods yielded larger values for $\Delta G_{b}^{e l}$ than the experimentally estimated values. Nevertheless, it should be noted that the experimentally estimated values of $\Delta G_{b}^{e l}$ could be in error if ionic strength has an effect on the structure of the proteins (Stone \& Hofsteenge, 1991). However, the values of $\Delta G_{b}^{e l}$ calculated were comparable with experimentally determined estimates for the total binding energy $\left(\Delta G_{b}\right)$. It is interesting to note that the value of $\Delta G_{b}^{e l}$ was relatively insensitive to variation in ionic strength from 0 to $0.125 \mathrm{M}$ in the MTK calculations but was found to vary about twofold experimentally and in the FD calculations.

\section{Decreases in binding energy caused by removal of negative charges from the C-terminal region of rhir}

Experimental values determined at an ionic strength of $0.125 \mathrm{M}$ are available for the change in binding energy $\left(\Delta \Delta G_{b}\right)$ resulting from the mutation of negatively charged residues in rhir. Estimates of the change in the electrostatic contribution to binding energy $\left(\Delta \Delta G_{b}^{e l}\right)$ were calculated by both the MTK and FD methods (Table 2). The values obtained on the basis of MTK calculations were generally in good agreement with the experimental results. The largest deviation was seen with the mutant D55N where the calculated value was about five times larger than the experimentally observed one. If this mutant is excluded, the average deviation between the experimental and calculated values was about $30 \%$. The plot of $\Delta \Delta G_{b}$ against $\Delta \Delta G_{b}^{e l}$ shown in Figure 4 illustrates the good agreement between the two values. The points do not deviate greatly from a line with a slope of 1 . The agreement between $\Delta \Delta G_{b}$ and $\Delta \Delta G_{b}^{e l}$ was even better if data obtained at the lower ionic strength of $0.05 \mathrm{M}$ for the mutants E62Q, E57,58Q, E57,58,62Q and E57,58, $61,62 \mathrm{Q}$ were compared with calculated values for this
Table 2. Comparison of the experimentally observed change in binding energy $\left(\Delta \Delta G_{b}\right)$ at an ionic strength of $0.125 \mathrm{M}$ with the change of the electrostatic binding energy calculated by the MTK $\left(\Delta \Delta G_{b}^{e l}(M T K)\right)$ and $F D\left(\Delta \Delta G_{b}^{e l}(F D)\right)$ methods ${ }^{\mathrm{a}}$

\begin{tabular}{lccr}
\hline & $\begin{array}{c}\Delta \Delta G_{b} \\
\left(\mathrm{~kJ} \mathrm{~mol}^{-1}\right)\end{array}$ & $\begin{array}{c}\Delta \Delta G_{b}^{\text {el }}(\mathrm{MTK}) \\
\left(\mathrm{kJ} \mathrm{mol}^{-1}\right)\end{array}$ & $\begin{array}{r}\Delta \Delta G_{b}^{\text {el }}(\mathrm{FD}) \\
\left(\mathrm{kJ} \mathrm{mol}^{-1}\right)\end{array}$ \\
\hline 1. D55N & 2.6 & $14.2(11.8)$ & $38.1(23.9)$ \\
2. E57Q & 5.9 & $2.8(-3.1)$ & $7.2(1.3)$ \\
3. E58Q & 5.3 & $3.9(-1.4)$ & $10.8(5.5)$ \\
4. E61Q & 1.3 & $0.6(-0.7)$ & $1.3(0.0)$ \\
5. E62Q & 2.8 & $2.3(-0.5)$ & $4.4(1.6)$ \\
6. E57,58Q & 6.0 & $8.6(2.6)$ & $18.2(12.2)$ \\
7. E61,62Q & 3.5 & $3.0(-0.5)$ & $5.7(2.2)$ \\
8. E57,58,62Q & 9.4 & $11.2(1.8)$ & $23.6(14.2)$ \\
9. E57,58,61,62Q & 10.6 & $12.4(1.8)$ & $25.2(14.6)$
\end{tabular}

${ }^{\mathrm{a}}$ The experimentally determined values for $\Delta \Delta G_{b}$ at $\mathrm{pH} 7.8$ and an ionic strength of $0.125 \mathrm{M}$ were taken from Braun et al. (1988a) and Betz et al. (1991). Values for $\Delta \Delta G_{b}^{e l}$ (MTK) and $\Delta \Delta G_{b}^{e l}$ (FD) were calculated as described under Methods of calculation. The deviation of the calculated from experimental value is given in parentheses. The number preceding each mutant identifies the data point for this mutant in Figure 4.

ionic strength (Table 3 ). The average deviation between the calculated and experimentally determined values for these mutants decreased from $30 \%$ at $0.125 \mathrm{M}$ ionic strength to $8 \%$ at $0.05 \mathrm{M}$.

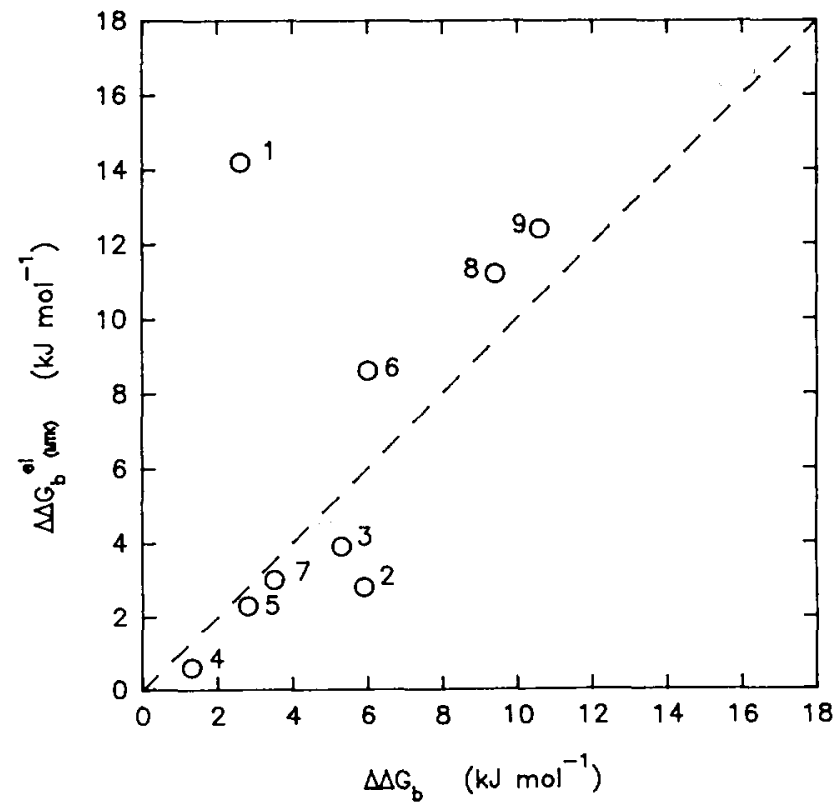

Fig. 4. Comparison of the experimentally observed change in binding energy $\left(\Delta \Delta G_{b}\right)$ at an ionic strength of $0.125 \mathrm{M}$ with the change of the electrostatic binding energy calculated by the MTK method $\left(\Delta \Delta G_{b}^{e l}\right.$ (MTK)). Data points for a particular mutant are identified by the number preceding the mutant in Table 2 . The values of $\Delta \Delta G_{b}$ and $\Delta \Delta G_{b}^{e l}$ (MTK) are taken from this table. 
Table 3. Comparison of the experimentally observed change in binding energy $\left(\Delta \Delta G_{b}\right)$ at an ionic strength of $0.05 \mathrm{M}$ with the change of the electrostatic binding energy calculated by the MTK method $\left(\Delta \Delta G_{b}^{e l}(M T K)\right)^{\mathrm{a}}$

\begin{tabular}{lcr}
\hline & $\begin{array}{c}\Delta \Delta G_{b} \\
\left(\mathrm{~kJ} \mathrm{~mol}^{-1}\right)\end{array}$ & $\begin{array}{c}\Delta \Delta G_{b}^{\text {el }}(\mathrm{MTK}) \\
\left(\mathrm{kJ} \mathrm{mol}^{-1}\right)\end{array}$ \\
\hline $\mathrm{E} 62 \mathrm{Q}$ & 2.8 & $2.4(-0.4)$ \\
$\mathrm{E} 57,58 \mathrm{Q}$ & 7.4 & $8.8(1.4)$ \\
$\mathrm{E} 57,58,62 \mathrm{Q}$ & 11.6 & $11.5(-0.1)$ \\
& 13.7 & $12.8(-0.9)$
\end{tabular}

${ }^{a}$ The experimentally determined values for $\Delta \Delta G_{b}$ at $\mathrm{pH} 7.8$ and an ionic strength of $0.05 \mathrm{M}$ were taken from Stone et al. (1989). Values for $\Delta \Delta G_{b}^{e l}$ (MTK) were calculated as described under Methods of calculation. The deviation of the calculated from experimental value is given in parentheses.

The agreement between $\Delta \Delta G_{b}$ and $\Delta \Delta G_{b}^{e l}$ was not as good when $\Delta \Delta G_{b}^{e l}$ was calculated using the FD method (Table 2). The deviation with the mutant D55 $\mathrm{N}$ was even larger than that observed with the MTK calculations. In addition, calculated values for forms of rhir in which Glu $58^{\prime}$ was mutated were all considerably higher than the experimentally observed values.

\section{Discussion}

Calculation of the electrostatic potential contours around thrombin and rhir indicated that the contours were asymmetric and complementary. The interaction of the complementary potentials would play a role in the stabilization of the complex. Decreases in binding energy were observed upon mutation of residues, such as Glu $61^{\prime}$ and Glu $62^{\prime}$, that are not directly involved in ionic interactions. The significant contribution to binding energy for the negative charges of these residues (Table 2) is a consequence of the interaction of the these charges with the positive electrostatic potential created by the fibrinogenrecognition exosite of thrombin. Structural alterations to thrombin that result in a decrease in the positive potential surrounding the fibrinogen-recognition exosite also reduce binding energy. $\gamma$-Thrombin is a proteolytically altered form of thrombin in which the 67-80-loop has been removed (Boissel et al., 1984). This structural modification will result in a marked reduction of the electrostatic potential of the fibrinogen-recognition exosite. The contribution of ionic interactions to binding energy is greatly reduced for the $\gamma$-thrombin-hirudin interaction (Stone \& Hofsteenge, 1991), and this result is consistent with an important role for the positive electrostatic potential of the fibrinogen-recognition site in the stabilization of the complex. In $\beta_{\mathrm{T}}$-thrombin, the 67-80-loop is cleaved, but it is not removed from the molecule as in $\gamma$-thrombin
(Braun et al., 1988b), and, consequently, the positive potential of the fibrinogen-recognition exosite should be basically preserved in $\beta_{\mathrm{T}}$-thrombin. As expected, the contribution of ionic interactions to binding energy with hirudin is also largely unaltered with $\beta_{\mathrm{T}}$-thrombin (Stone \& Hofsteenge, 1991).

In addition to their role in the stabilization of the complex, the complementarity of the electrostatic potentials (and fields) will play an important role in the kinetics of complex formation. Charged molecules should diffuse together more rapidly than neutral molecules. In addition, the complementarity of the electrostatic potentials will preorientate thrombin and rhir such that productive complex formation will be encouraged. This hypothesis is supported by experimental data. The association rate constant for rhir was markedly reduced by mutations that removed negative charges from the $\mathrm{C}$-terminal region of hirudin and, thus, reduced the strength of the negative potential around this region of the molecule (Stone et al., 1989). Indeed, the rate-limiting step in the formation of the complex appears to be the electrostatic interaction between the C-terminal tail of hirudin and the fibrinogenrecognition exosite (Stone \& Hofsteenge, 1986). This phenomenon known as "electrostatic steering" has been postulated to be important in the association of other molecules (Matthew, 1985; Klapper et al., 1986; Sharp et al., 1987; Northrup et al., 1988; Tulinsky et al., 1988).

In most cases, MTK calculations successfully predicted the changes in binding energy caused by a particular mutation; in a plot of $\Delta \Delta G_{b}$ against $\Delta \Delta G_{b}^{e l}$, the points did not in general deviate significantly from a line with a slope of 1 (Fig. 4). These results suggest that for most mutations only the electrostatic part of the binding energy was changed by the mutation and that nonpolar interactions have not been significantly altered. These observations are consistent with the results of a previous study that indicated that the nonionic interactions were not affected for mutations involving C-terminal glutamyl residues (Stone et al., 1989). The agreement between $\Delta \Delta G_{b}$ and $\Delta \Delta G_{b}^{e l}$ was even better when data obtained at lower ionic strength were considered (Table 3 ). This result is not surprising because the Debye-Hückel approximation was used to calculate the ionic strength dependence of the electrostatic interactions, and this approximation is only valid for low ionic strengths.

The largest discrepancy between $\Delta \Delta G_{b}$ and $\Delta \Delta G_{b}^{e l}$ was seen for the mutant D55N. In the complex of rhir with thrombin, Asp 55' forms salt bridges with Arg 73 and Lys 149E. The method of calculation of $\Delta \Delta G_{b}^{e l}$ may have led to an overestimation of this parameter. It was assumed that the structure of free thrombin was the same as that of complexed thrombin. Comparison of the structure of thrombin in complex with rhir and D-Phe-Pro$\mathrm{ArgCH}_{2}$-thrombin indicates that this is in general true with the root mean square (rms) deviation of polypeptide backbone atoms of thrombin being only $0.45 \AA$ (Bode 
et al., 1989; Rydel et al., 1990, 1991). However, the rms deviation for the 146-150-loop, in which Lys $149 \mathrm{E}$ is located, is $4.9 \AA$. The susceptibility of this loop to protease cleavage suggests that it is quite flexible (Berliner, 1984; Brezniak et al., 1990), and crystallography studies suggest that it is held in one conformation only by crystal-packing restraints (Rydel et al., 1991; SkrzypczakJankun et al., 1991). Thus, the conformation of this loop in the crystal structure of the thrombin-rhir complex probably does not represent its structure either in unbound thrombin or in the complex in solution. The calculation of the free energy of unbound thrombin assumed that Arg 73 and Lys 149E are located close to each other as observed in the complex, whereas the flexibility of the 145-150-loop could allow Lys 149E to move away and avoid the unfavorable contact. If this unfavorable contact does not occur, the contribution to binding energy of the electrostatic interaction with Asp 55' will be overestimated. Other evidence suggests that the calculations may have overestimated the strength of the salt bridge between Asp 55' and Lys 149E. This salt bridge is not found in the complex of thrombin with hirugen, a peptide based on the C-terminal sequence of hirudin (SkrzypczakJankun et al., 1991). Thus, at least for the thrombinhirudin interaction, this Lys $149 \mathrm{E}-\mathrm{Asp} 55^{\prime}$ interaction appears not to play an important role. Moreover, the $\Delta \Delta G_{b}$ for the interaction of the mutant D55N with bovine thrombin is indistinguishable from that obtained with human thrombin (A. Betz \& S.R. Stone, unpubl.). In bovine thrombin, the residue corresponding to Lys $149 \mathrm{E}$ is a glutamate and, thus, unable to form a salt bridge with Asp 55'. These two observations suggest that the salt bridge between Lys 149E and Asp 55' does not make a significant contribution to binding energy. Nevertheless, the remaining salt bridge to $\mathrm{Arg} 73$ would be expected to contribute more than $2.6 \mathrm{~kJ} \mathrm{~mol}^{-1}$ to binding energy. Site-specific mutants of thrombin are required to resolve this apparent inconsistency.

The agreement between $\Delta \Delta G_{b}$ and $\Delta \Delta G_{b}^{e l}$ was not as close when $\Delta \Delta G_{b}^{e l}$ was calculated by the FD instead of the MTK method (Table 2). The discrepancy between $\Delta \Delta G_{b}$ and $\Delta \Delta G_{b}^{e l}$ for the mutant D55N was even larger than observed with the MTK calculations and large discrepancies were also observed when forms of rhir in which Glu $58^{\prime}$ was mutated were considered (Table 2). In all cases, $\Delta \Delta G_{b}^{e l}$ overestimates $\Delta \Delta G_{b}$. Both Asp 55' and Glu $58^{\prime}$ are involved in close electrostatic interactions, and it seems possible that the FD method overestimates the free energy of such interactions. Figure 5 is a plot of the electrostatic free energy of all inter- and intramolecular charge-charge interactions in the thrombin-rhir complex as calculated by the MTK and FD methods against the distance between the charges. The FD method provides higher values for the short range charge-charge interactions than the MTK method. Overestimation of short-range electrostatic effects has been observed in

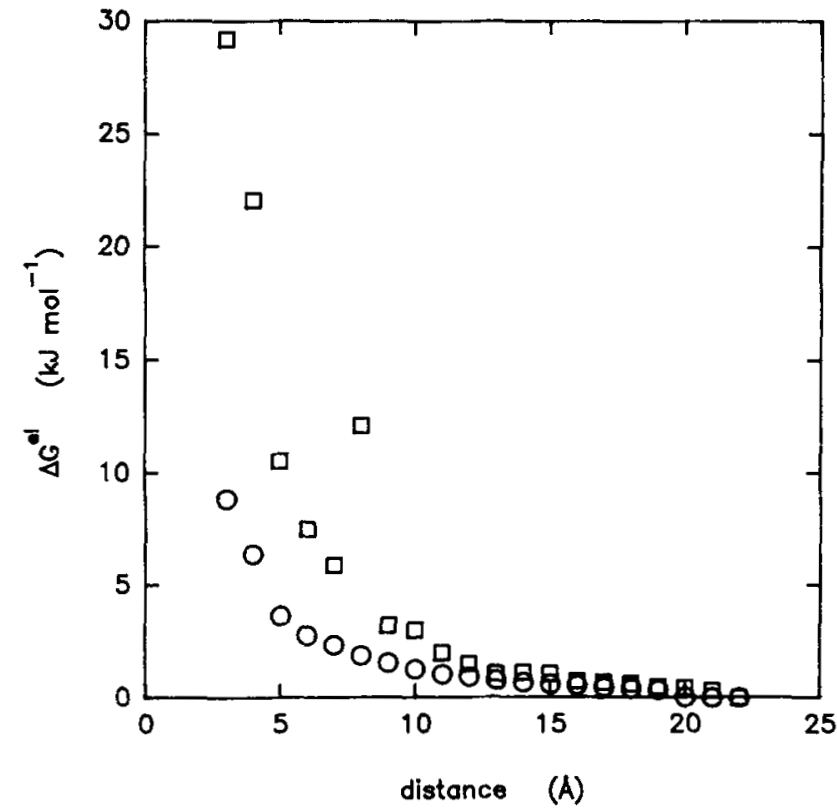

Fig. 5. Distance dependence of the electrostatic interaction energy $\left(\Delta G^{e l}\right.$ ) as calculated by the MTK and FD methods. The estimates of $\Delta G^{e l}$ as calculated by the MTK $(0)$ and FD ( $(0)$ methods are plotted against the separation distance of the two charges. Data were calculated from every pair of interacting charges in the thrombin-rhir complex and averaged for a distance segment of $1 \AA$. For example, the absolute values of the energy calculated for charges separated between $2.5 \AA$ and $3.5 \AA$ were averaged and are given by point at $3 \AA$. The fluctuation of the $\Delta G^{\text {el }}$ at $8 \AA$ (FD calculation) is due to the small sample between 7.5 and $8.5 \AA$.

other studies in which the $\mathrm{p} K_{a}$ values of Cys 247 of rhodanese (Gilson \& Honig, 1988) and Asp 158 of papain (Menard et al., 1990) were calculated. Gilson et al. (1987) have noted that the inaccuracy of the FD calculations increases in the vicinity of the charges due to grid representation of the system. In an attempt to improve the accuracy of the FD calculations, the grid size for the complex was reduced from $1.2 \AA$ to $1.0 \AA$ A during the second focusing step for calculation of $\Delta \Delta G_{b}^{e l}$ for several mutants. This reduced grid size did not result in an improvement of the agreement between $\Delta \Delta G_{b}^{e l}$ and $\Delta \Delta G_{b}$ in all cases; the average change in $\Delta \Delta G_{b}^{e l}$ was only $0.5 \mathrm{~kJ} \mathrm{~mol}^{-1}$. Thus, given the relative uncertainty of the structure of the C-terminal tail of uncomplexed hirudin (see below), we felt that the accuracy of the estimates of $\Delta \Delta G_{b}^{e l}$ would not be improved by decreasing the grid size further. Another possible cause of the overestimation of $\Delta \Delta G_{b}^{e l}$ is the representation of the protein dielectric that is used in the FD calculations. It has been pointed out by Warshel and Åvist (1991) that the charge groups are usually found in a polar environment even when they are buried in the protein. This corresponds to an increase in the local dielectric constant and will cause a decrease in the strength of charge-charge interactions. Thus, a more detailed de- 
scription of the protein dielectric constant may improve the theoretical results. We are currently attempting to develop an algorithm for FD calculations that will allow the mapping of the dielectric constant inside the protein. In MTK calculations, a factor linearly proportional to the solvent accessibility of the charged groups was used (Karshikov et al., 1989), and this leads to a decrease in the interaction energy.

An additional possible reason for the discrepancies between $\Delta \Delta G_{b}$ and $\Delta \Delta G_{b}^{e l}$ for mutants involving Glu $58^{\prime}$ is that the crystal structure does not correctly describe the structure in this region due to intermolecular crystal contacts. The salt bridge between Glu 58' and Arg 77A is not found in the structure of thrombin with hirugen (Skrzypczak-Jankun et al., 1991). In addition, Glu 57' forms a salt bridge with Arg 75 of a neighboring molecule in the crystal (Rydel et al., 1990, 1991; SkrzypczakJankun et al., 1991) and this interaction may distort the structure of this region. Moreover, the effects of mutating Glu 57' and Glu 58' were not found to be additive, which suggests that a conformational change or change of mechanism may be involved in the binding of these two residues (Betz et al., 1991).

Although the general agreement between $\Delta \Delta G_{b}$ and $\Delta \Delta G_{b}^{e l}$ was quite good (particularly for MTK calculations), both MTK and FD calculations overestimate the contribution of electrostatic interactions to binding energy $\left(\Delta \Delta G_{b}^{e l}\right.$; Table 1). As noted previously, the experimentally estimated values of $\Delta G_{b}^{e l}$ could be in error if ionic strength has an effect on the structure of the proteins (Stone et al., 1991). However, the estimates for $\Delta G_{b}^{e l}$ were about equal to the experimentally determined value for total binding energy $\left(\Delta G_{b}\right)$, and it seems unlikely that the contribution of electrostatic interactions is as high as suggested by the results of the calculations. The large number of nonpolar contacts between thrombin and rhir are expected to make a considerable contribution to binding energy (Rydel et al., 1990, 1991). One of the reasons for the overestimation of the value of $\Delta G_{b}^{e l}$ could be due to the assumptions made with respect to the structures of free thrombin and rhir. The problems with the structure of the regions of thrombin interacting with Asp $55^{\prime}$ and Glu 57'/Glu 58' have been dealt with above. The atomic coordinates for the complexed rhir were used in the calculation of the electrostatic interactions for the free molecule. If the conformation of rhir in solution is substantially different from that of bound rhir, the calculation may have over- or underestimated the electrostatic term of the free energy of the unbound rhir. Values for $\Delta G_{h}^{e l}$ of about 0 (MTK) or $3.8 \mathrm{~kJ} \mathrm{~mol}^{-1}$ (FD) were obtained. These values indicate negligible or unfavorable electrostatic interactions in free rhir. However, the rhir molecule may have another conformation in solution with more optimized electrostatic interactions. Indeed, the solution structure of hirudin as determined by twodimensional NMR techniques indicates that the C-termi- nal region of hirudin does not have a defined structure in solution (Folkers et al., 1989; Haruyama \& Wüthrich, 1989), which contrasts to the well-ordered structure of this region in the complex.

Thus, problems with the definition of the solution structures of thrombin, rhir, and the complex are most likely the cause of the difficulties in calculating a good estimate for $\Delta G_{b}^{e l}$. In spite of these difficulties, however, the MTK method has proven generally reliable in estimating the decreases in binding energy due to mutation of the charged residues. With two residues (Glu 61' and Glu $\left.62^{\prime}\right)$, these decreases in binding energy could not have been readily predicted from examination of the crystal structure. Thus, the results of the present study indicate that interpretation of the effects of mutating charged residues can be facilitated by simple electrostatic calculations using the MTK method.

\section{Method of calculations}

FD calculations were performed using the appropriate programs of the DELPHI package (Klapper et al., 1986; Gilson \& Honig, 1988). The calculations were started with Coulombic boundary conditions (Klapper et al., 1986) followed by two-step focusing runs. The final calculations used a $65 \times 65 \times 65$ grid with the length of each cell being $1.0 \AA, 0.9 \AA$, and $1.2 \AA$ for thrombin, rhir, and the complex, respectively.

MTK calculations were performed as described by Karshikov et al. (1989). In this case, the charge values were not determined a priori. The $\mathrm{p} K$ values of the titratable groups were calculated (Karshikov et al., 1989), and then the charges on these groups were determined from the $\mathrm{p} K$ values. The intrinsic $\mathrm{p} K$ values used in these calculations were taken from Matthew (1985).

\section{Application to the interaction of thrombin and rhir}

The coordinates of the crystal structure of the thrombinrhir complex determined by Rydel et al. (1990) were used in all calculations. Thrombin and rhir were assumed to have the same structures in their uncomplexed states as in the complex. Dielectric constants of 78 and 4 were assigned to the solvent and to the protein microphase, respectively, in both MTK and FD calculations. The radii of the dielectric cavities of the components required in MTK calculations were determined to be $27 \AA$ for the complex, $24 \AA$ for thrombin, and $18 \AA$ for rhir. In both computational approaches, the charges carried by the titratable groups were treated as point charges whose coordinates were calculated as described previously (Karshikov et al., 1989). The calculations assumed a pH of 7.8. Ionic strength was taken into account by using the DebyeHückel approximation (Karshikov et al., 1989). 
Calculations of the electrostatic contribution to binding energy

The electrostatic term of the binding energy of the thrombin-rhir complex, $\Delta G_{b}^{e l}$, was calculated according to the formula

$$
\Delta G_{b}^{e l}=\Delta G_{t-h}^{e l}-\left(\Delta G_{t}^{e l}+\Delta G_{h}^{e l}\right),
$$

where the subscripts $t-h, t$, and $h$ designate the electrostatic term of free energy of the thrombin-rhir complex, thrombin, and rhir, respectively. The electrostatic term of free energy is defined as the work required to move each charge, $q_{i}$, from an infinite distance to its position in the field of all other, $j$, charges:

$$
\Delta G^{e l}=\frac{1}{2} \sum_{i \neq j} q_{i} \Phi_{j},
$$

where $\Phi_{j}$ is the electrostatic potential created by the $j$ charges. Values of $\Delta G_{t-h}^{e l}, \Delta G_{t}^{e l}$, and $\Delta G_{h}^{e l}$ were calculated using Equation 2. The change of the electrostatic term of the binding energy upon mutation of charged groups of rhir is given by

$$
\begin{aligned}
\Delta \Delta G_{b}^{e l} & =\Delta G_{b}^{e l}-\Delta G_{b}^{e l^{\prime}} \\
& =\Delta G_{t-h}^{e l}-\Delta G_{t-h}^{e l^{\prime}}-\Delta G_{h}^{e l}+\Delta G_{h}^{e l^{\prime}},
\end{aligned}
$$

where the prime designates the free energy for the mutated rhir. Due to the structural similarity of the mutated side chains (only Asp $\rightarrow$ Asn and Glu $\rightarrow$ Gln were considered), the charge set of the mutated rhir could be simply represented by deletion of the charge of the mutated residue for the calculation of $\Delta G_{t-h}^{e l^{\prime}}$ and $\Delta G_{h}^{e l^{\prime}}$.

In order to save computing time in the case of FD calculations, the symmetry of Equation 2 was used, and the electrostatic interaction energy of all charges in the field of the mutated residue was calculated. Thus, the change in the electrostatic term of binding energy will be given by Equation 4:

$$
\begin{aligned}
\Delta \Delta G^{e l}= & \sum_{k}^{l} \Phi_{k}^{t-h} \sum_{i}^{N_{Q}} Q_{i}+\sum_{k}^{l}\left(\Phi_{k}^{t-h}-\Phi_{k}^{h}\right) \sum_{i}^{n^{\prime}} q_{i} \\
& +\sum_{k \neq m}^{l}\left(\Phi_{k}^{t-h}-\Phi_{k}^{h}\right) \sum_{m}^{l} q_{m},
\end{aligned}
$$

where $\Phi_{k}^{t-h}$ and $\Phi_{k}^{h}$ are the potentials created by the charges that disappear due to mutation calculated for the complex and free hirudin, respectively, $l$ is the number of mutations, $Q_{i}$ and $q_{i}$ are charges on thrombin and hirudin, respectively, and $q_{m}$ are the mutated charges. The total number of charges on thrombin is $N_{Q}$, whereas the corresponding value for hirudin is $l+n^{\prime}$. The first term in the above equation corresponds to the change in the interactive electrostatic energy between the partners, and the last two reflect the change in the solvation energy due to the change in the charge composition of the system. If only one charge group is mutated $(l=1)$, Equation 4 reduces to

$$
\Delta \Delta G^{e l}=\Phi_{m u t}^{t-h} \sum_{i}^{N_{Q}} Q_{i}+\left(\Phi_{m u t}^{t-h}-\Phi_{m u t}^{h}\right) \sum_{i}^{n^{\prime}} q_{i} .
$$

\section{Acknowledgments}

We thank Prof. R. Huber for his support and encouragement and Dr. A.M. Lesk for his helpful comments. This work was supported by NIH grant HL43229 (A.T.) and the Sonderforschungsbereich 207 of the Universität München (W.B.).

\section{References}

Bashford, D. (1991). Electrostatic effects in biological molecules. Curr. Opin. Struct. Biol. I, 175-184.

Berliner, L.J. (1984). Structure-function relationships in human $\alpha$-and $\gamma$-thrombin. Mol. Cell. Biochem. 61, 159-172.

Betz, A., Hofsteenge, J., \& Stone, S.R. (1991). Role of ionic interactions in the formation of the thrombin-hirudin complex. Biochem. J. 275, 801-803.

Bode, W., Huber, R., Rydel, T.J., \& Tulinsky, A. (1991). X-ray crystal structures of human $\alpha$-thrombin and of the human $\alpha$-thrombinhirudin complex. In Thrombin: Structure and Function (Berliner, L.J., Ed.). Plenum, New York (in press).

Bode, W., Mayr, I., Baumann, U., Huber, R., Stone, S.R., \& Hofsteenge, J. (1989). The refined $1.9 \AA$ crystal structure of human $\alpha$-thrombin: Interaction with D-Phe-Pro-Arg chloromethylketone and significance of the Tyr-Pro-Pro-Trp insertion loop. EMBO J. $8,3467-3475$.

Bode, W., Turk, D., \& Karshikov, A. (1992). The refined 1.9-^ X-ray crystal structure of D-Phe-Pro-Arg chloromethylketone-inhibited human $\alpha$-thrombin: Structure analysis, overall structure, electrostatic properties, detailed active-site geometry, and structure-function relationships. Protein Sci. 1, 426-471.

Boissel, J.-P., Le Bonniec, B., Rabiet, M.-J., Labie, D., \& Elion, J. (1984). Covalent structures of $\beta$ and $\gamma$ autolytic derivatives of human $\alpha$-thrombin. J. Biol. Chem. 259, 5691-5697.

Braun, P.J., Dennis, S., Hofsteenge, J., \& Stone, S.R. (1988a). Use of site-directed mutagenesis to investigate the basis for the specificity of hirudin. Biochemistry 27, 6517-6522.

Braun, P.J., Hofsteenge, J., Chang, J.-Y., \& Stone, S.R. (1988b). Preparation of characterization of proteolysed forms of human $\alpha$-thrombin. Thromb. Res. 50, 273-283.

Brezniak, D.V., Brower, M.S., Witting, J.I., Walz, D.A., \& Fenton, J.W., II (1990). Human $\alpha$-to $\zeta$-thrombin cleavage occurs with neutrophil cathepsin $\mathrm{G}$ or chymotrypsin while fibrinogen clotting activity is retained. Biochemistry 29, 3526-3542.

Fenton, J.W., II (1988). Regulation of thrombin generation and functions. Semin. Thromb. Hemostasis 14, 234-240.

Folkers, P.J.M., Clore, G.M., Driscoll, P.C., Dodt, J., Kohler, S., \& Gronenborn, A.M. (1989). Solution structure of recombinant hirudin and the Lys47-Glu mutant: A nuclear magnetic resonance and hybrid geometry-dynamical simulated annealing study. Biochemistry 28, 2601-2617.

Gilson, M.K. \& Honig, B.H. (1988). Energetics of charge-charge interactions in proteins. Proteins 3, 32-53.

Gilson, M.K., Sharp, K.A., \& Honig, B.H. (1987). Calculating electrostatic interactions in bio-molecules: Method and error assessment J. Comput. Chem. 9, 237-335.

Grütter, M.G., Priestle, J.P., Rahuel, J., Grossenbacher, H., Bode, W., Hof steenge, J., \& Stone, S.R. (1990). Crystal structure of the thrombin-hirudin complex: A novel mode of serine protease inhibition. $E M B O$ J. 9, 2361-2365.

Haruyama, H. \& Wüthrich, K. (1989). Conformation of recombinant 
desulfatohirudin in aqueous solution determined by nuclear magnetic resonance. Biochemistry 28, 4301-4312.

Karshikov, A., Engh, R., Bode, W., \& Atanasov, B. (1989). Electrostatic interactions in proteins: Calculations of the electrostatic term of free energy and the electrostatic potential field. Eur. J. Biophys. $17,287-297$.

Kirkwood, J.G. (1934). Theory of solutions of molecules containing widely separated charges with spatial application to zwitterions. J. Chem. Phys. 2, 351-361.

Klapper, I., Hagstrom, R., Fine, R., Sharp, K., \& Honig, B. (1986). Focussing of electric field in the active site of $\mathrm{Cu}-\mathrm{Zn}$ superoxide dismutase: Effect of ionic strength and amino acid modification. Proteins $1,47-59$.

Matthew, J.B. (1985). Electrostatic effects in proteins. Annu. Rev. Biophys. Biophys. Chem. 14, 387-417.

Matthew, J.B. \& Gurd, F.R.N. (1986). Calculation of electrostatic interactions in proteins. Methods Enzymol. 130, 413-453.

Menard, R., Khouri, H.E., Plouffe, C., Dupars, R., Ripoll, D., Vernet, T., Tessier, D.C., Laliberte, F., Thomas, D.Y., \& Storer, A.S. (1990). A protein engineering study of the role of aspartate 158 in the catalytic mechanism of papain. Biochemistry $29,6706-6713$.

Northrup, S., Boles, J., \& Reynolds, J. (1988). Brownian dynamics of cytochrome $\mathrm{c}$ and cytochrome $\mathrm{c}$ peroxidase association. Science 241 , 67-70.

Rydel, T.J., Ravichandran, K.G., Tulinsky, A., Bode, W., Huber, R., Roitsch, C., \& Fenton, J.W., II (1990). The structure of a complex of recombinant hirudin and human $\alpha$-thrombin. Science 249 , 277-280.

Rydel, T.J., Tulinsky, A., Bode, W., \& Huber, R. (1991). Refined structure of the hirudin-thrombin complex. J. Mol. Biol. 221, 583-601.

Sharp, K., Fine, R., \& Honig, B. (1987). Computer simulations of the diffusion of a substrate to an active site of an enzyme. Science 236, 1460-1463.

Sharp, K. \& Honig, B. (1990). Calculation of total electrostatic energy with the nonlinear Poisson-Boltzmann equation. J. Phys. Chem. 94 7684-7692.

Shire, S.J., Hanania, G.I.H., \& Gurd, F.R.N. (1974). Electrostatic effect in myoglobin. Hydrogen ion equilibria in sperm whale ferrimyoglobin. Biochemistry 13, 2967-2974.

Skrzypczak-Jankun, E., Carperos, V.E., Ravichandran, K.G., Tulinsky, A., Westbrook, M., \& Maraganore, J.M. (1991). The structures of the hirugen and hirulog I complexes of $\alpha$-thrombin. J. Mol. Biol. $221,1379-1393$

Stone, S.R., Dennis, S., \& Hofsteenge, J. (1989). Quantitative evaluation of the contribution of ionic interactions to the formation of the thrombin-hirudin complex. Biochemistry 28, 6857-6863.

Stone, S.R. \& Hofsteenge, J. (1986). Kinetics of inhibition of thrombin by hirudin. Biochemistry $25,4622-4628$.

Stone, S.R. \& Hofsteenge, J. (1991). Basis for the reduced affinity of human $\beta_{\mathrm{T}^{-}}$and $\gamma_{\mathrm{T}^{-}}$thrombin for hirudin. Biochemistry 30,3950 3955.

Tanford, C. \& Kirkwood, J.G. (1957). Theory of protein titration curves. I. General equation for impenetrable spheres. J. Am. Chem. Soc. $79,5333-5339$.

Tulinsky, A., Park, C.H., Mao, B., \& Llinas, M. (1988). Lysine/fibrin binding sites of kringles modeled after the structure of kringle I of prothrombin. Proteins 3, 85-96.

Warshel, A. \& Åqvist, J. (1991). Electrostatic energy and macromolecular function. Annu. Rev. Biophys. Biophys. Chem. 20, 267-298.

Warwicker, J. \& Watson, H.C. (1982). Calculation of the electric potential in the size cleft due to $\alpha$-helix dipoles. J. Mol. Biol. 157, 671-679. 